PROCEEDINGS OF THE

AMERICAN MATHEMATICAL SOCIETY

Volume 134, Number 12, December 2006, Pages 3665-3675

S 0002-9939(06)08453-X

Article electronically published on May 31, 2006

\title{
ON THE MINIMUM OF SEVERAL RANDOM VARIABLES
}

\author{
Y. GORDON, A. E. LITVAK, C. SCHÜTT, AND E. WERNER
}

(Communicated by N. Tomczak-Jaegermann)

\begin{abstract}
For a given sequence of real numbers $a_{1}, \ldots, a_{n}$, we denote the $k$ th smallest one by $k$ - $\min _{1 \leq i \leq n} a_{i}$. Let $\mathcal{A}$ be a class of random variables satisfying certain distribution conditions (the class contains $N(0,1)$ Gaussian random variables). We show that there exist two absolute positive constants $c$ and $C$ such that for every sequence of real numbers $0<x_{1} \leq \ldots \leq x_{n}$ and every $k \leq n$, one has

$$
c \max _{1 \leq j \leq k} \frac{k+1-j}{\sum_{i=j}^{n} 1 / x_{i}} \leq \mathbb{E} k-\min _{1 \leq i \leq n}\left|x_{i} \xi_{i}\right| \leq C \ln (k+1) \max _{1 \leq j \leq k} \frac{k+1-j}{\sum_{i=j}^{n} 1 / x_{i}},
$$

where $\xi_{1}, \ldots, \xi_{n}$ are independent random variables from the class $\mathcal{A}$. Moreover, if $k=1$, then the left-hand side estimate does not require independence of the $\xi_{i}$ 's. We provide similar estimates for the moments of $k-\min _{1 \leq i \leq n}\left|x_{i} \xi_{i}\right|$ as well.
\end{abstract}

\section{INTRODUCTION}

For a given sequence of real numbers $a_{1}, \ldots, a_{n}$, we denote the $k$ th smallest one by $k-\min _{1 \leq i \leq n} a_{i}$; thus, $1-\min _{1 \leq i \leq n} a_{i}=\min _{1 \leq i \leq n} a_{i}$, and $2-\min _{1 \leq i \leq n} a_{i}$ is the next smallest, etc., and $\left(k-\min _{1 \leq i \leq n} a_{i}\right)_{k=1}^{n}$ is the nondecreasing rearrangement of the sequence $\left(a_{i}\right)_{i=1}^{n}$. In the same way, we denote the $k$ th biggest number by $k$ - $\max _{1 \leq i \leq n} a_{i}$.

In the paper [GLSW1] we considered expressions of the form

$$
\mathbb{E} \sum_{k=1}^{m} k-\max _{1 \leq i \leq n}\left|x_{i} f_{i}\right|^{p}
$$

where $f_{1}, f_{2}, \ldots, f_{n}$ are random variables and $x_{1}, x_{2}, \ldots, x_{n}$ are real numbers. Since the functions $\left(\sum_{k=1}^{m} k-\max _{1 \leq i \leq n}\left|x_{i}\right|^{p}\right)^{1 / p}$ are norms on $\mathbb{R}^{n}$, such forms appear naturally in the study of various parameters associated with the geometry of Banach spaces [GLSW2. Other applications of these forms can be found in [KS1] and KS2].

Received by the editors March 7, 2005 and, in revised form, June 25, 2005.

2000 Mathematics Subject Classification. Primary 62G30, 60E15, 60G51.

Key words and phrases. Order statistics, expectations, moments, normal distribution, exponential distribution.

The first author was partially supported by the Fund for the Promotion of Research at the Technion and by France-Israel Cooperation agreement \#3-1350.

The first and third authors were partially supported by FP6 Marie Curie Actions, MRTN-CT2004-511953, PHD.

The fourth author was partially supported by an NSF Grant, by a Nato Collaborative Linkage Grant, and by an NSF Advance Opportunity Grant. 
The striking difference in the present study is that we now consider expressions of the form $\left(\sum_{k \in I} k \text { - } \min _{1 \leq i \leq n}\left|x_{i}\right|^{p}\right)^{1 / p}$ for subsets $I \subseteq\{1, \ldots, n\}$. These are not norms if $I$ is not an integer interval starting at 1 . Hence, for a given sequence of random variables $f_{1}, \ldots, f_{n}$, the computation of expressions such as

$$
\mathbb{E} k-\min _{1 \leq i \leq n}\left|f_{i}\right|^{p}=\mathbb{E}(n-k+1)-\max _{1 \leq i \leq n}\left|f_{i}\right|^{p}
$$

requires completely different techniques. Such minima, also called order statistics, have been intensively studied during last century. We refer an interested reader to $\mathrm{AB}$ and $\mathrm{DN}$ for basic facts, known results, and references. Most works dealt with the case of independent identically distributed random variables. Sometimes the condition "to be identically distributed" was substituted by the condition "the $f_{i}$ 's have the same first and the same second moments". In this paper we drop these conditions and deal with sequences of random variables having no restrictions on their moments. Applications of the current paper are related to important electrical engineering problems on the minimization of the data loss of signals emanating from electrical networks. Applications appear also in the study of the multifold $K$-functional or its geometric equivalent, the norm with unit ball $B=\operatorname{co}\left(\bigcup_{i=1}^{m} B_{i}\right)$, where the $B_{i}$ 's are unit balls of symmetric normed spaces.

Now we describe our setting and results. Let $\alpha>0, \beta>0$ be parameters. We say that a random variable $\xi$ satisfies the $(\alpha, \beta)$-condition if

$$
\mathbb{P}(|\xi| \leq t) \leq \alpha t \quad \text { for every } t \geq 0
$$

and

$$
\mathbb{P}(|\xi|>t) \leq e^{-\beta t} \quad \text { for every } t \geq 0 .
$$

Note that this forces the condition $\alpha t+e^{-\beta t} \geq 1$ for all $t \geq 0$, which implies $\alpha \geq \beta$.

Below (Claim 1 and the remark following it) we will see that many random variables, including $N(0,1)$ Gaussian variables (with $\alpha=\beta=\sqrt{2 / \pi}$ ) and exponentially distributed variables (with $\alpha=\beta=1$ ), satisfy this condition. We study first the moments of the minimum of a sequence of such random variables. Let $x_{1}, \ldots, x_{n}$ be a sequence of real numbers and $\xi_{1}, \ldots, \xi_{n}$ be independent random variables satisfying the $(\alpha, \beta)$-condition. We obtain that for every $p>0$, one has

$$
\frac{1}{1+p} \alpha^{-p}\left(\sum_{i=1}^{n} \frac{1}{\left|x_{i}\right|}\right)^{-p} \leq \mathbb{E} \min _{1 \leq i \leq n}\left|x_{i} \xi_{i}\right|^{p} \leq \beta^{-p} \Gamma(1+p)\left(\sum_{i=1}^{n} \frac{1}{\left|x_{i}\right|}\right)^{-p}
$$

where $\Gamma(\cdot)$ is the Gamma-function. Moreover, the left-hand side estimate does not require the independence of the $\xi_{i}$ 's. In particular, it implies that for every $p>0$,

$$
\mathbb{E} \min _{1 \leq i \leq n}\left|x_{i} g_{i}\right|^{p} \leq \Gamma(2+p) \mathbb{E} \min _{1 \leq i \leq n}\left|x_{i} f_{i}\right|^{p},
$$

where $g_{1}, \ldots, g_{n}$ are independent $N(0,1)$ Gaussian random variables and $f_{1}, \ldots, f_{n}$ are $N(0,1)$ Gaussian random variables (not necessarily independent). Taking $p=1$, we have

$$
\mathbb{E} \min _{1 \leq i \leq n}\left|x_{i} g_{i}\right| \leq 2 \mathbb{E} \min _{1 \leq i \leq n}\left|x_{i} f_{i}\right|
$$

This result should be compared with the well-known Šdák's inequality ([S], G] $)$, saying that

$$
\mathbb{E} \max _{1 \leq i \leq n}\left|x_{i} g_{i}\right| \geq \mathbb{E} \max _{1 \leq i \leq n}\left|x_{i} f_{i}\right|
$$


In other words, our result is, in a sense, an inverse Šidák's inequality. It would be nice to eliminate the factor 2 from it.

We generalize our estimates for the expectation of the minimum to the case of the $k$ th minimum. For $0<x_{1} \leq x_{2} \leq \ldots \leq x_{n}$ and independent random variables $\xi_{1}, \ldots, \xi_{n}$ satisfying the $(\alpha, \beta)$-condition, we obtain that there are two absolute positive constants $c$ and $C$ such that for every $p>0$, one has

$$
\begin{aligned}
c_{p} \alpha^{-1} \max _{1 \leq j \leq k} \frac{k+1-j}{\sum_{i=j}^{n} 1 / x_{i}} & \leq\left(\mathbb{E} k-\min _{1 \leq i \leq n}\left|x_{i} \xi_{i}\right|^{p}\right)^{1 / p} \\
& \leq C(p, k) \beta^{-1} \max _{1 \leq j \leq k} \frac{k+1-j}{\sum_{i=j}^{n} 1 / x_{i}}
\end{aligned}
$$

where $c_{p}=c^{1+1 / p}$ and $C(p, k)=C \max \{p, \ln (k+1)\}$. We would like to emphasize that the estimates are pretty sharp, in particular, the ratio of upper and lower bounds surprisingly does not depend on $n$ and, up to a constant depending only on $p$, is bounded by $\ln (k+1)$.

The latter result implies that we may evaluate sums of the form

$$
\sum_{k \in I} \mathbb{E} k-\min _{1 \leq i \leq n}\left|x_{i} g_{i}\right|^{p}
$$

where $I \subset\{1,2, \ldots, n\}$ is any subset of integers and the $g_{i}$ 's are $N(0,1)$ Gaussian random variables.

The paper is organized as follows: In Section 2, we introduce the notation, quote some known facts, and prove that random variables with certain densities, including Gaussian and exponential, satisfy the $(\alpha, \beta)$-condition. In Section 3 we provide combinatorial results used in the proofs. In Section 4 , we prove our main theorems. Finally, in Section 5, we provide some examples.

An extended abstract of this work appeared in [GLSW3].

\section{NotATION AND PRELIMINARIES}

Given $A \subset \mathbb{N}$ we denote its cardinality by $|A|$. Given a set $E$ we denote its complement by $E^{c}$. We say that $\left(A_{j}\right)_{j=1}^{k}$ is a partition of $\{1,2, \ldots, n\}$ if $\emptyset \neq A_{j} \subset$ $\{1,2, \ldots, n\}, j \leq k, \bigcup_{j \leq k} A_{j}=\{1,2, \ldots, n\}$, and $A_{i} \cap A_{j}=\emptyset$ for $i \neq j$. The canonical Euclidean norm and the canonical inner product on $\mathbb{R}^{n}$ we denote by $|\cdot|$ and $\langle\cdot, \cdot\rangle$. By $1 / t$ we mean $\infty$ if $t=0$ and 0 if $t=\infty$.

As we defined above, $\left(k \text { - } \min _{1 \leq i \leq n} a_{i}\right)_{k=1}^{n}$ denotes the nondecreasing rearrangement of the sequence $\left(a_{i}\right)_{i=1}^{n}$, i.e., $k$ - $\min \left(a_{i}\right)_{i=1}^{n}$ is the $k$ th smallest element of the sequence.

We will use the following simple properties of $k$-min which hold for every sequence $\left(a_{i}\right)_{i=1}^{n}$.

For every $r<k$,

$$
k-\min \left(a_{i}\right)_{i=1}^{n} \geq(k-r)-\min \left(a_{i}\right)_{i=r+1}^{n} .
$$

For every partition $\left(A_{j}\right)_{j \leq k}$ of $\{1,2, \ldots, n\}$,

$$
k-\min \left(a_{i}\right)_{i=1}^{n} \leq \max _{j \leq k}\left\{\min _{i \in A_{j}} a_{i}\right\}_{j \leq k} .
$$

Now we recall some definitions and estimates connected to the Gaussian distribution. 
Let $x$ and $y$ be nonnegative real numbers. The Gamma-function is defined by

$$
\Gamma(x)=\int_{0}^{\infty} t^{x-1} e^{-t} d t .
$$

Note that

$$
x \Gamma(x)=\Gamma(1+x), \quad \Gamma(n+1)=n !,
$$

and, by Stirling's formula, for every $x \geq 1$,

$$
\sqrt{2 \pi x}\left(\frac{x}{e}\right)^{x}<\Gamma(x+1)<\sqrt{2 \pi x}\left(\frac{x}{e}\right)^{x} e^{\frac{1}{12 x}} .
$$

Finally, we show examples of random variables, satisfying the $(\alpha, \beta)$-condition.

Claim 1. Let $q \geq 1$. Let $\xi$ be a nonnegative random variable with the density function $p(s)=c_{q} \exp \left(-s^{q}\right)$, where $c_{q}=1 / \Gamma(1+1 / q)$. Then $\xi$ satisfies (1) and (2) with parameters $\alpha=\beta=c_{q}$.

Remark. An important case is the case $q=2$ which corresponds to the Gaussian random variable. Claim 1 implies that $N(0,1)$ Gaussian random variables satisfy the $(\alpha, \beta)$-condition with $\alpha=\beta=\sqrt{2 / \pi}$. We would also like to note that if $q=1$, then we have an exponentially distributed random variable. In this case $\alpha=\beta=1$.

Proof of Claim 1. The case $q=1$ is trivial. So we assume that $q>1$. Clearly, we have

$$
\mathbb{P}(|\xi| \leq t)=c_{q} \int_{0}^{t} e^{-s^{q}} d s \leq c_{q} t
$$

which shows $\alpha=c_{q}$.

Now consider the function $g$ defined on $[0, \infty)$ by

$$
g(x)=\exp \left(-c_{q} x\right)-c_{q} \int_{x}^{\infty} e^{-s^{q}} d s
$$

Then $g(0)=\lim _{x \rightarrow \infty} g(x)=0$ and $g^{\prime}(x)=c_{q}\left(\exp \left(-x^{q}\right)-\exp \left(-c_{q} x\right)\right)$. Hence, $g^{\prime}(x) \geq 0$ on $\left[0, c_{q}^{1 /(q-1)}\right]$ and $g^{\prime}(x) \leq 0$ for $x \geq c_{q}^{1 /(q-1)}$. It shows that $g(x) \geq 0$ for every $x \geq 0$. Therefore,

$$
\mathbb{P}(|\xi|>t)=c_{q} \int_{t}^{\infty} e^{-s^{q}} d s \leq e^{-c_{q} t}
$$

i.e., $\beta=c_{q}$.

\section{Combinatorial Results}

In this section we prove some combinatorial results, which will be used later in the proofs of theorems.

First we quote the following result on symmetric means ([HLP).

Lemma 2. Let $1 \leq l \leq n$. Let $a_{i}, i=1, \ldots, n$, be nonnegative real numbers. Then

$$
\sum_{\substack{A \subset\{1,2, \ldots, n\} \\
|A|=l}} \prod_{i \in A} a_{i} \leq\left(\begin{array}{c}
n \\
l
\end{array}\right)\left(\frac{1}{n} \sum_{i=1}^{n} a_{i}\right)^{l} .
$$

We will need the following consequence of this lemma. 
Corollary 3. Let $1 \leq k \leq n$. Let $a_{i}, i=1, \ldots, n$, be nonnegative real numbers. Assume

$$
0<a:=\frac{e}{k} \sum_{i=1}^{n} a_{i}<1
$$

Then

$$
\sum_{l=k}^{n} \sum_{\substack{A \subset\{1,2, \ldots, n\} \\|A|=l}} \prod_{i \in A} a_{i}<\frac{1}{\sqrt{2 \pi k}} \frac{a^{k}}{1-a} .
$$

Proof. By Lemma 2 we have

$$
\sum_{l=k}^{n} \sum_{\substack{A \subset\{1,2, \ldots, n\} \\
|A|=l}} \prod_{i \in A} a_{i} \leq \sum_{l=k}^{n}\left(\begin{array}{l}
n \\
l
\end{array}\right)\left(\frac{k a}{e n}\right)^{l}=\sum_{l=k}^{n} \frac{n ! a^{l} k^{l}}{l !(n-l) ! e^{l} n^{l}} \leq \sum_{l=k}^{n} \frac{a^{l} k^{l}}{l ! e^{l}} .
$$

Applying Stirling's formula (5), we obtain

$$
\sum_{l=k}^{n} \sum_{\substack{A \subset\{1,2, \ldots, n\} \\|A|=l}} \prod_{i \in A} a_{i} \leq \sum_{l=k}^{n} \frac{a^{l} k^{l}}{l^{l} \sqrt{2 \pi l}} \leq \frac{1}{\sqrt{2 \pi k}} \sum_{l=k}^{n} a^{l}<\frac{1}{\sqrt{2 \pi k}} \frac{a^{k}}{1-a} .
$$

We will also need the following result.

Lemma 4. Let $1 \leq k \leq n$. Let $\left(a_{i}\right)_{i=1}^{n}$ be a nonincreasing sequence of positive real numbers. Then there exists a partition $\left(A_{l}\right)_{l \leq k}$ of $\{1,2, \ldots, n\}$ such that

$$
\min _{1 \leq l \leq k} \sum_{i \in A_{l}} a_{i} \geq a:=\frac{1}{2} \min _{1 \leq j \leq k} \frac{1}{k+1-j} \sum_{i=j}^{n} a_{i} .
$$

Remark. In fact, our proof gives that the $A_{l}$ 's can be taken as intervals, i.e., $A_{l}=$ $\left\{i \mid n_{l-1}<i \leq n_{l}\right\}, l \leq k$, for some sequence $0=n_{0}<1 \leq n_{1}<n_{2}<\ldots<n_{k}=n$.

Proof. Denote $b:=\sum_{i=1}^{n} a_{i}$.

Case 1. $a_{1} \leq b / k$. Let $n_{0}=0$ and, given $1 \leq l \leq k$, let $n_{l}$ be the largest integer such that

$$
\sum_{i=1}^{n_{l}} a_{i} \leq \frac{l b}{k}
$$

Since $b / k \geq a_{1} \geq a_{2} \geq \ldots \geq a_{n}$, we have $0=n_{0}<1 \leq n_{1}<n_{2}<\ldots<n_{k}=n$. Define a partition $\left(A_{l}\right)_{l \leq k}$ of $\{1,2, \ldots, n\}$ by $A_{l}=\left\{i \mid n_{l-1}<i \leq n_{l}\right\}$. Let $t$ be the largest integer such that $a_{t}>\frac{b}{2 k}$ (if there is no such $a_{t}$ we put $t=0$ ). Then

[i] for every $l$ such that $n_{l} \leq t$ we have $\sum_{i \in A_{l}} a_{i} \geq a_{n_{l}}>\frac{b}{2 k}$;

[ii] for every $l<k$ such that $n_{l}>t$ we have $\sum_{i \in A_{l}} a_{i} \geq \frac{b}{2 k}$ (otherwise, since $a_{n_{l}+1} \leq \frac{b}{2 k}$, we would have

$$
\sum_{i=1}^{n_{l}+1} a_{i} \leq \sum_{i=1}^{n_{l-1}} a_{i}+\sum_{i \in A_{l}} a_{i}+a_{n_{l}+1}<\frac{(l-1) b}{k}+\frac{b}{2 k}+\frac{b}{2 k}=\frac{l b}{k},
$$

which contradicts the choice of $n_{l}$ );

[iii] for $l=k$ we have $\sum_{i \in A_{k}} a_{i}=\sum_{i=1}^{n} a_{i}-\sum_{i=1}^{n_{k-1}} a_{i} \geq \frac{b}{k}$. 
Since $\frac{b}{2 k} \geq a$, this proves the result in Case 1 .

Case 2. $a_{1}>b / k$. Denote $b_{j}:=\sum_{i=j}^{n} a_{i}, j \leq n$. Clearly, $a_{k} \leq b_{k}$. Let $m \leq k$ be the smallest integer such that

$$
a_{m} \leq \frac{b_{m}}{k+1-m} .
$$

Since $a_{1}>b / k=b_{1} / k$, we have $m>1$. For $1 \leq l<m$ choose $A_{l}=\{l\}$. Then

$$
\sum_{i \in A_{l}} a_{i}=a_{l}>\frac{b_{l}}{k+1-l}>a .
$$

Let $\left(A_{l}\right)_{l=m}^{k}$ be the partition of $\{m, m+1, \ldots, n\}$ into $k+1-m$ sets constructed in the same way as in Case 1 . Then, by Case 1 , for every $l \geq m$,

$$
\sum_{i \in A_{l}} a_{i} \geq \frac{b_{m}}{2(k+1-m)} \geq a .
$$

This completes the proof.

Remark. One can show that the sequence

$$
\bar{a}_{j}=\frac{1}{k+1-j} \sum_{i=j}^{n} a_{i}
$$

$j \leq k$, considered in the last lemma (and which will appear again below) has the following properties: There exists an integer $1 \leq r \leq k$ such that

$$
\bar{a}_{1}>\bar{a}_{2}>\ldots>\bar{a}_{r} \leq \bar{a}_{r+1} \leq \ldots \leq \bar{a}_{k}
$$

and

$$
\bar{a}_{j+1} \geq \bar{a}_{j} \quad \text { if and only if } \sum_{i=j+1}^{n} a_{i} \geq(k-j) a_{j} .
$$

To see (7), note that by the formula for $\bar{a}_{j}$ 's we immediately obtain that $\bar{a}_{j+1} \geq \bar{a}_{j}$ if and only if

$$
(k+1-j) \sum_{i=j+1}^{n} a_{i} \geq(k-j) \sum_{i=j}^{n} a_{i}=(k-j) \sum_{i=j+1}^{n} a_{i}+(k-j) a_{j},
$$

which implies (7). Now note that (7) implies that

$$
\text { if } \quad \bar{a}_{j+1} \geq \bar{a}_{j}, \quad \text { then } \quad \bar{a}_{j+2} \geq \bar{a}_{j+1} .
$$

Indeed, if $\bar{a}_{j+1} \geq \bar{a}_{j}$, then, by (77), $(k-j) a_{j} \leq \sum_{i=j+1}^{n} a_{i}$. Therefore, since $\left(a_{i}\right)_{i}$ is nonincreasing,

$$
(k-j-1) a_{j+1} \leq(k-j) a_{j}-a_{j+1} \leq \sum_{i=j+1}^{n} a_{i}-a_{j+1}=\sum_{i=j+2}^{n} a_{i},
$$

which implies $\bar{a}_{j+2} \geq \bar{a}_{j+1}$ by (7). Finally, (6) is a simple consequence of (8). 


\section{MAin Results}

In this section we state our main theorems, discussed in the introduction, and provide corresponding deviation inequalities which imply the main theorems.

Theorem 5. Let $\alpha>0, \beta>0$. Let $p>0$. Let $\left(x_{i}\right)_{i=1}^{n}$ be a sequence of real numbers and let $\xi_{1}, \ldots, \xi_{n}$ be random variables satisfying the $(\alpha, \beta)$-condition. Then

$$
\frac{1}{1+p} \alpha^{-p}\left(\sum_{i=1}^{n} 1 /\left|x_{i}\right|\right)^{-p} \leq \mathbb{E} \min _{1 \leq i \leq n}\left|x_{i} \xi_{i}\right|^{p} .
$$

Moreover, if the $\xi_{i}$ 's are independent, then

$$
\mathbb{E} \min _{1 \leq i \leq n}\left|x_{i} \xi_{i}\right|^{p} \leq \beta^{-p} \Gamma(1+p)\left(\sum_{i=1}^{n} 1 /\left|x_{i}\right|\right)^{-p} .
$$

An immediate consequence of this theorem is the following corollary.

Corollary 6. Let $p>0$. Let $\left(x_{i}\right)_{i=1}^{n}$ be a sequence of real numbers and let $f_{1}, \ldots, f_{n}, \xi_{1}, \ldots, \xi_{n}$ be random variables satisfying the $(\alpha, \beta)$-condition. Assume that the $\xi_{i}$ 's are independent. Then

$$
\mathbb{E} \min _{1 \leq i \leq n}\left|x_{i} \xi_{i}\right|^{p} \leq \Gamma(2+p) \alpha^{p} \beta^{-p} \mathbb{E} \min _{1 \leq i \leq n}\left|x_{i} f_{i}\right|^{p} .
$$

In particular, if $f_{1}, \ldots, f_{n}, \xi_{1}, \ldots, \xi_{n}$ are $N(0,1)$ Gaussian random variables, then

$$
\mathbb{E} \min _{1 \leq i \leq n}\left|x_{i} \xi_{i}\right|^{p} \leq \Gamma(2+p) \mathbb{E} \min _{1 \leq i \leq n}\left|x_{i} f_{i}\right|^{p} .
$$

Theorem 7. Let $\alpha>0, \beta>0$. Let $p>0$ and $2 \leq k \leq n$. Let $0<x_{1} \leq$ $x_{2} \leq \ldots \leq x_{n}$ and let $\xi_{1}, \ldots, \xi_{n}$ be independent random variables satisfying the $(\alpha, \beta)$-condition. Then

$c_{p \alpha} \max _{1 \leq j \leq k} \frac{k+1-j}{\sum_{i=j}^{n} 1 / x_{i}} \leq\left(\mathbb{E} k-\min _{1 \leq i \leq n}\left|x_{i} \xi_{i}\right|^{p}\right)^{1 / p} \leq \beta^{-1} C(p, k) \max _{1 \leq j \leq k} \frac{k+1-j}{\sum_{i=j}^{n} 1 / x_{i}}$,

where $c_{p \alpha}=\frac{1}{2 e \alpha}\left(1-\frac{1}{4 \sqrt{\pi}}\right)^{1 / p}$ and $C(p, k)=4 \sqrt{2} \max \{p, \ln (1+k)\}$.

Theorems 5 and 7 are consequences of the following lemmas, which are of independent interest. In GLSW3 we showed how these lemmas imply the theorems in the Gaussian case. Since the proof of the general case, which we consider here, is done in exactly the same way, we omit the proofs of the theorems and concentrate on the proofs of the lemmas.

Lemma 8. Let $\alpha>0, \beta>0$. Let $0<x_{1} \leq x_{2} \leq \ldots \leq x_{n}$ and let $\xi_{1}, \ldots, \xi_{n}$ be random variables satisfying the $(\alpha, \beta)$-condition. Let $a=\sum_{i=1}^{n} 1 / x_{i}$. Then for every $t>0$,

$$
\mathbb{P}\left\{\omega\left|\min _{1 \leq i \leq n}\right| x_{i} \xi_{i}(\omega) \mid \leq t\right\} \leq \alpha a t .
$$

Moreover, if the $\xi_{i}$ 's are independent, then for every $t>0$,

$$
\mathbb{P}\left\{\omega\left|\min _{1 \leq i \leq n}\right| x_{i} \xi_{i}(\omega) \mid>t\right\} \leq e^{-\beta a t}
$$


Proof. Denote $A_{k}(t)=\left\{\omega|| x_{k} \xi_{k}(\omega) \mid>t\right\}=\left\{\omega|| \xi_{k}(\omega) \mid>t / x_{k}\right\}$ and

$$
A(t)=\left\{\omega\left|\min _{k \leq n}\right| x_{k} \xi_{k}(\omega) \mid>t\right\}=\bigcap_{k \leq n} A_{k}(t) .
$$

By (11) we have $\mathbb{P}\left(A_{k}(t)^{c}\right) \leq \alpha t / x_{k}$. Therefore,

$$
\mathbb{P}(A(t)) \geq 1-\sum_{k=1}^{n} \mathbb{P}\left(A_{k}(t)^{c}\right) \geq 1-\alpha t \sum_{k=1}^{n} 1 / x_{k},
$$

which proves the first estimate.

Now assume that the $\xi_{k}$ 's are independent. By (2) we have

$$
\mathbb{P}\left(A_{k}(t)\right) \leq \exp \left(-\beta t / x_{k}\right) .
$$

Therefore,

$$
\mathbb{P}(A(t))=\prod_{k=1}^{n} \mathbb{P}\left(A_{k}(t)\right) \leq \exp \left(-\beta \sum_{k=1}^{n} t / x_{k}\right),
$$

which proves the result.

Lemma 9. Let $\alpha>0, \beta>0$. Let $1 \leq k \leq n$. Let $0<x_{1} \leq x_{2} \leq \ldots \leq x_{n}$ and let $\xi_{1}, \ldots, \xi_{n}$ be independent random variables satisfying the $(\alpha, \beta)$-condition. Let

$$
a=\frac{\alpha e}{k} \sum_{i=1}^{n} \frac{1}{x_{i}}
$$

Then for every $0<t<1 / a$, one has

$$
\mathbb{P}\left\{\omega\left|k-\min _{1 \leq i \leq n}\right| x_{i} \xi_{i}(\omega) \mid \leq t\right\} \leq \frac{1}{\sqrt{2 \pi k}} \frac{(a t)^{k}}{1-a t} .
$$

Proof. Denote $A(t)=\mathbb{P}\left\{\omega\left|k-\min _{1 \leq i \leq n}\right| x_{i} \xi_{i}(\omega) \mid \leq t\right\}$. Clearly, we have

$$
\begin{aligned}
A(t) & =\mathbb{P}\left\{\omega\left|\exists i_{1}, \ldots, i_{k} \geq 1:\right| \xi_{i_{j}}(\omega) \mid \leq \frac{t}{x_{i_{j}}}\right\} \\
& =\mathbb{P} \bigcup_{\ell=k}^{n} \bigcup_{\substack{A \subset\{1, \ldots, n\} \\
|A|=\ell}}\left\{\omega|\forall i \in A:| \xi_{i}(\omega) \mid \leq \frac{t}{x_{i}} \text { and } \forall i \notin A:\left|\xi_{i}(\omega)\right|>\frac{t}{x_{i}}\right\} \\
& =\sum_{l=k}^{n} \sum_{\substack{A \subset\{1, \ldots, n\} \\
|A|=i}} \prod_{i \in A} \mathbb{P}\left\{\omega|| \xi_{i}(\omega) \mid \leq \frac{t}{x_{i}}\right\} \prod_{i \notin A} \mathbb{P}\left\{\omega|| \xi_{i}(\omega) \mid>\frac{t}{x_{i}}\right\} .
\end{aligned}
$$

It follows that

$$
A(t) \leq \sum_{l=k}^{n} \sum_{\substack{A \subset\{1, \ldots, n\} \\|A|=l}} \prod_{i \in A} \mathbb{P}\left\{\omega|| \xi_{i}(\omega) \mid \leq \frac{t}{x_{i}}\right\} \leq \sum_{l=k}^{n} \sum_{\substack{A \subset\{1, \ldots, n\} \\|A|=l}} \prod_{i \in A} \alpha \frac{t}{x_{i}} .
$$

Corollary 3 implies the desired result. 


\section{EXAMPLES}

In this section we provide some examples. They show that Theorem 7 is sharp. Namely, we show that both, the upper and lower estimate in Theorem 7 can be attained. We also provide an example where the actual value of $\mathbb{E} k$-min lies between the two estimates.

Example 10. Let $1 \leq k \leq n$. Let $g_{i}, i \leq n$, be independent $N(0,1)$ Gaussian random variables. Then:

(i) For $k \leq n / 2$,

$$
\sqrt{\frac{\pi}{2}} \frac{k}{n+1} \leq \mathbb{E} k-\min _{1 \leq i \leq n}\left|g_{i}\right| \leq \sqrt{2 \pi} \frac{k}{n+1} .
$$

(ii) For $k \geq n / 2$,

$$
c \sqrt{\ln \frac{2 n}{n+1-k}} \leq \mathbb{E} k_{-} \min _{1 \leq i \leq n}\left|g_{i}\right| \leq C \sqrt{\ln \frac{2 n}{n+1-k}},
$$

where $c$ and $C$ are absolute positive constants.

Remark. Note that in this case $x_{1}=\cdots=x_{n}=1$, hence $\max _{1 \leq j \leq k} \frac{k+1-j}{\sum_{i=j}^{n} 1 / x_{i}}=$ $k / n$. Thus (i) of this example shows that the lower estimate in Theorem 7 can be attained (up to an absolute constant).

Proof of Example 10, (i) follows immediately from the following claim:

Claim. For all $1 \leq k \leq n$ one has

$$
\sqrt{\frac{\pi}{2}} \frac{k}{n+1} \leq \mathbb{E} k-\min _{1 \leq i \leq n}\left|g_{i}\right| \leq \sqrt{\frac{\pi}{2}} \frac{k}{n+1-k} .
$$

We now prove the claim. Denote $u=u(t)=\mathbb{P}\left\{\left|g_{1}\right|>t\right\}=\sqrt{\frac{2}{\pi}} \int_{t}^{\infty} e^{-s^{2} / 2} d s$. Using the same ideas as in the proof of Lemma 9, we observe that

$$
\begin{aligned}
\mathbb{E} k-\min _{1 \leq i \leq n}\left|g_{i}\right| & =\int_{0}^{\infty} \mathbb{P}\left\{k-\min _{1 \leq i \leq n}\left|g_{i}\right|>t\right\} d t \\
& =\sum_{l=0}^{k-1} \sum_{\substack{A \subset\{1, \ldots, n\} \\
|A|=n-l}} \int_{0}^{\infty}\left(\prod_{i \in A} u(t) \prod_{i \notin A}(1-u(t))\right) d t \\
& =\sum_{l=0}^{k-1}\left(\begin{array}{c}
n \\
l
\end{array}\right) \int_{0}^{\infty}(u(t))^{n-l}(1-u(t))^{l} d t \\
& =\sqrt{\frac{\pi}{2}} \sum_{l=0}^{k-1}\left(\begin{array}{c}
n \\
l
\end{array}\right) \int_{0}^{\infty}(u(t))^{n-l}(1-u(t))^{l} e^{t^{2} / 2}(-d u(t)) .
\end{aligned}
$$

To obtain the lower estimate note that $e^{t^{2} / 2} \geq 1$ and, therefore,

$$
\mathbb{E} k-\min _{1 \leq i \leq n}\left|g_{i}\right| \geq \sqrt{\frac{\pi}{2}} \sum_{l=0}^{k-1}\left(\begin{array}{l}
n \\
l
\end{array}\right) B(n-l+1, l+1)=\sqrt{\frac{\pi}{2}} \frac{k}{n+1},
$$

where $B(x, y)=\int_{0}^{1} s^{x-1}(1-s)^{y-1} d s=\Gamma(x) \Gamma(y) / \Gamma(x+y)$ is the Beta-function. 
To obtain the upper estimate note that by considering the function $f(t)=$ $e^{t^{2} / 2} \int_{x}^{\infty} e^{-s^{2} / 2} d s$ one can obtain that $u(t) e^{t^{2} / 2} \leq 1$ and, therefore,

$\mathbb{E} k-\min _{1 \leq i \leq n}\left|g_{i}\right| \leq \sqrt{\frac{\pi}{2}} \sum_{l=0}^{k-1}\left(\begin{array}{l}n \\ l\end{array}\right) B(n-l, l+1)=\sqrt{\frac{\pi}{2}} \sum_{l=0}^{k-1} \frac{1}{n-l} \leq \sqrt{\frac{\pi}{2}} \frac{k}{n+1-k}$.

(ii) is well known.

The next two examples can be obtained by direct calculation as well. One should split a sequence into two parts and use deviation inequalities. We omit the details.

Example 11. Let $1 \leq k \leq n / 2$. Let $g_{i}, i \leq n$, be independent exponentially distributed random variables (i.e. with density $p(s)=e^{-s}$ ). Let $x_{1}=\cdots=x_{k}=1$, $x_{k+1}=\cdots=x_{n}=n^{2}$. Then

$$
c \ln k \leq \mathbb{E} k-\min _{1 \leq i \leq n}\left|x_{i} g_{i}\right| \leq C \ln k,
$$

where $c$ and $C$ are absolute positive constants.

Remark. Note that in this case $\max _{1 \leq j \leq k} \frac{k+1-j}{\sum_{i=j}^{n} 1 / x_{i}}=\frac{k}{k+(n-k) / n^{2}} \approx 1$. Thus, this example shows that the upper estimate in Theorem 7 can be attained (up to an absolute constant).

Example 12. Let $1 \leq k \leq n$. Let $g_{i}, i \leq n$, be independent $N(0,1)$ Gaussian random variables. Let $x_{1}=\cdots=x_{k}=1, x_{k+1}=\cdots=x_{n}=n^{2}$. Then

$$
c \sqrt{\ln k} \leq \mathbb{E} k-\min _{1 \leq i \leq n}\left|x_{i} g_{i}\right| \leq C \sqrt{\ln k}
$$

where $c$ and $C$ are absolute positive constants.

\section{REFERENCES}

[AB] B. C. ARnold, N. Balakrishnan Relations, bounds and approximations for order statistics, Lecture Notes in Statistics, 53, Berlin etc.: Springer-Verlag. viii, 1989. MR0996887(90i:62061)

[DN] H. A. David, H. N. Nagaraja, Order statistics, third ed., Wiley Series in Probability and Statistics. Chichester: John Wiley \& Sons, 2003. MR1994955 (2004f:62007)

[G] E. D. GLuskin, Extremal properties of orthogonal parallelepipeds and their applications to the geometry of Banach spaces, Math. USSR Sbornik, 64 (1989), 85-96. MR0945901 (89j:46016)

[GLSW1] Y. Gordon, A. E. Litvak, C. Schütt, E. Werner, Orlicz norms of sequences of random variables, Ann. of Prob., 30 (2002), 1833-1853. MR.1944007(2003m:60009)

[GLSW2] Y. Gordon, A. E. Litvak, C. Schütt, E. Werner, Geometry of spaces between zonoids and polytopes, Bull. Sci. Math., 126 (2002), 733-762. MR.1941083(2003i:52006)

[GLSW3] Y. Gordon, A. E. Litvak, C. Schütt, E. Werner, Minima of sequences of Gaussian random variables, C. R. Acad. Sci. Paris, Sér. I Math., 340 (2005), 445-448. MR 2135327

[HLP] G. H. Hardy, J. E. Littlewood and G. Polya, Inequalities, second ed., Cambridge, The University Press. XII, 1952. MR0046395 (13:727e)

[KS1] S. KWAPIEN, C. SCHÜtT, Some combinatorial and probabilistic inequalities and their application to Banach space theory, Studia Math. 82 (1985), 91-106. MR0809774 (87h:46042) 
[KS2] S. KwapIEn, C. SсHÜtT, Some combinatorial and probabilistic inequalities and their application to Banach space theory. II, Studia Math. 95 (1989), 141-154. MR1038501 (91e:46027)

[S] Z. S̆IDÁK, Rectangular confidence regions for the means of multivariate normal distributions, J. Am. Stat. Assoc. 62 (1967), 626-633. MR0216666 (35:7495)

Department of Mathematics, Technion, Haifa 32000, Israel

E-mail address: gordon@techunix.technion.ac.il

Department of Mathematics and Statistics Sciences, University of Alberta, Edmonton, Alberta, Canada T6G 2G1

E-mail address: alexandr@math.ualberta.ca

Mathematisches Seminar, Christian Albrechts Universität, 24098 Kiel, Germany

E-mail address: schuett@math.uni-kiel.de

Department of Mathematics, Case Western Reserve University, Cleveland, Ohio 44106 and Université de Lille 1, UFR de Mathématique, 59655 Villeneuve D'AscQ, France

E-mail address: emw2@po.cwru.edu 